\title{
On the Projective Ricci Curvature
}

\author{
Zhongmin Shen* and Liling Sun ${ }^{\dagger}$
}

February 24, 2020

\begin{abstract}
The notion of the Ricci curvature is defined for sprays on a manifold. With a volume form on a manifold, every spray can be deformed to a projective spray. The Ricci curvature of a projective spray is called the projective Ricci curvature. In this paper, we introduce the notion of projectively Ricci-flat sprays. We establish a global rigidity result for projectively Ricci-flat sprays with nonnegative Ricci curvature. Then we study and characterize projectively Ricci-flat Randers metrics.

Keywords: spray, Finsler metric, Randers metric, projective Ricci curvature.
\end{abstract}

Mathematics Subject Classification 2010: 53B40, 53C60

\section{Introduction}

In Finsler geometry, there are many important Riemannian quantities such as the Riemann curvature and the Ricci curvature, etc. and non-Riemannian quantities such as the Berwald curvature and the S-curvature, etc. The Ricci curvature is defined as the trace of the Riemann curvature. Together with the S-curvature, the Ricci curvature plays an important role in Finsler geometry. The volume of geodesic balls can be controlled by the lower bounds of the Ricci curvature and the S-curvature (3, 4]). The volume of geodesic balls can be also controlled by a single bound of the N-Ricci curvature which is the combination of the Ricci curvature and the S-curvature ([1]).

Let $\mathbf{G}$ be a spray on an $n$-dimensional manifold $M$. Given a volume form $d V$ on $M$, we can construct a new spray by

$$
\hat{\mathbf{G}}:=\mathbf{G}+\frac{2 \mathbf{S}}{n+1} \mathbf{Y} .
$$

The spray $\hat{\mathbf{G}}$ is called the projective spray of $(\mathbf{G}, d V)$. The projective Ricci curvature of $(\mathbf{G}, d V)$ is defined as the Ricci curvature of $\hat{\mathbf{G}}$, namely,

$$
\operatorname{PRic}_{(G, d V)}:=\mathbf{R i c}_{\hat{G}} .
$$

\footnotetext{
*supported in part by NSF China (NSFC No. 11671352)
}

${ }^{\dagger}$ corresponding author 
By a simple computation, we have the following formula for the projective Ricci curvature:

$$
\operatorname{PRic}_{(G, d V)}=\mathbf{R i c}+(n-1)\left\{\frac{\mathbf{S}_{\mid 0}}{n+1}+\left[\frac{\mathbf{S}}{n+1}\right]^{2}\right\} .
$$

where $\mathbf{R i c}=\mathbf{R i c}_{G}$ is the Ricci curvature of the spray $\mathbf{G}, \mathbf{S}=\mathbf{S}_{(G, d V)}$ is the S-curvature of $(\mathbf{G}, d V)$ and $\mathbf{S}_{\mid 0}$ is the covariant derivative of $\mathbf{S}$ along a geodesic of $\mathbf{G}$. It is known that $\hat{\mathbf{G}}$ remains unchanged under a projective change of $\mathbf{G}$ with $d V$ fixed, thus $\mathbf{P R i c}_{(\mathbf{G}, d V)}=\mathbf{R i c}_{\hat{\mathbf{G}}}$ is a projective invariant of $(\mathbf{G}, d V)$ (2]). We make the following

Definition 1.1 A spray $\mathbf{G}$ on an $n$-dimensional manifold $M$ is said to be projectively Ricci-flat if there is a volume form $d V$ on $M$ such that

$$
\operatorname{PRic}_{(G, d V)}=0 .
$$

A Finsler metric $F$ on $M$ is said to be projectively Ricci-flat if the induced spray $\mathbf{G}=\mathbf{G}_{F}$ is projectively Ricci-flat.

It is easy to see that every projectively flat spray on $R^{n}$ is projectively Ricciflat. For projectively equivalent sprays, if one of them is projectively Ricci-flat, then so is the other.

We prove the following

Theorem 1.2 Let $\mathbf{G}$ be a spray on an n-dimensional manifold $M . \mathbf{G}$ is projectively Ricci-flat if and only if there are a volume form $d V$ and a scalar function $f$ on $M$ such that

$$
\mathbf{R i c}_{G}=-(n-1)\left\{\Xi_{\mid 0}+\Xi^{2}\right\}
$$

where "|" is the horizontal covariant derivative with respect to $\mathbf{G}, f_{0}=f_{x^{m}} y^{m}$, $\Xi:=\frac{\mathbf{S}}{n+1}-f_{0}$ and $\mathbf{S}=\mathbf{S}_{(G, d V)}$.

If the spray satisfies that $\mathbf{S}_{(G, d V)}=(n+1) \phi_{0}$ for some scalar function $\phi$ on $M$, then $\Xi=0$ for $f=\phi$. We obtain the following

Corollary 1.3 Let $\mathbf{G}$ be a spray on a manifold $M$. Suppose that $\mathbf{R i c}_{G}=0$ and $\mathbf{S}_{(G, d V)}=(n+1) \phi_{0}$ for some volume form $d V$ and scalar function $\phi$ on $M$, then $\mathbf{G}$ is projectively Ricci-flat.

The condition on the S-curvature, $\mathbf{S}_{(G, d V)}=(n+1) \phi_{0}$ being an exact 1-form, is actually a condition on the spray, independent of the choice of a particular volume form $d V$. Sprays with this property have vanishing $\chi$-curvature $\chi=0$ ([5], Proposition 4.1). We have the following rigidity theorem:

Theorem 1.4 Let $\mathbf{G}$ be a complete spray on an $n$-manifold $M$. Suppose that $\mathbf{G}$ is projectively Ricci-flat. If the Ricci curvature $\mathbf{R i c}_{G} \geq 0$, then for any volume form $d V$ on $M$, the $S$-curvature $\mathbf{S}=(n+1) \phi_{0}$ for some scalar function $\phi$ on $M$. In this case, $\mathbf{R i c}_{G}=0$. 
To have a better understanding on projectively Ricci-flat Finsler metrics, we consider a Randers metric $F=\alpha+\beta$ on an $n$-dimensional manifold $M$, where $\alpha=\sqrt{a_{i j} y^{i} y^{j}}$ is a Riemannian metric and $\beta=b_{i} y^{i}$ is a 1 -form with $\|\beta\|_{\alpha}<1$. Put

$$
s_{i j}:=\frac{1}{2}\left(b_{i ; j}-b_{j ; i}\right),
$$

where ";" denotes the covariant derivative with respect to Levi-Civita connection of $\alpha$. Clearly, $\beta$ is closed if and only if $s_{i j}=0$. We prove the following

Theorem 1.5 Let $F=\alpha+\beta$ be a Randers metric on an n-dimensional manifold M.F is projectively Ricci-flat if and only if there is a scalar function $h$ on $M$ such that

$$
\begin{aligned}
& \mathbf{R i c}_{\alpha}=2 s_{0 m} s_{0}^{m}+\alpha^{2} s^{i}{ }_{j} s_{i}-(n-1)\left[h_{0 ; 0}+\left(h_{0}\right)^{2}\right] \\
& s^{m}{ }_{0 ; m}=(n-1) h_{x^{m}} s^{m}{ }_{0},
\end{aligned}
$$

where $\mathbf{R i c}_{\alpha}$ denotes the Ricci curvature of $\alpha$.

In general, projectively Ricci-flat Randers metrics are not Ricci-flat, and the S-curvature is not almost isotropic.

In [7, Cheng-Shen-Ma study the projective Ricci curvature PRic. They derive a formula for the projective Ricci curvature of a Randers metric with respect to the Busemann-Hausdorff volume form $d V_{B H}$. By this formula, they characterize Randers metrics with $\mathbf{R R i c}=0$ with respect to $d V_{B H}$. We should point out that the projective Ricci-flatness of Randers metrics defined in [7] is slightly different from ours. Thus the statement of Theorem 1.1 in [7] is slightly different from Theorem 1.5 .

\section{Preliminaries}

Let $M$ be an $n$-dimensional manifold and $T M$ the corresponding tangent bundle. We denote by $T M_{0}=T M \backslash\{0\}$ the slit tangent bundle. Local coordinates on the base manifold $M$ will be denoted by $\left(x^{i}\right)$, while the induced local coordinates on $T M$ or $T M_{0}$ will be denoted by $\left(x^{i}, y^{i}\right)$. We call $\left(x^{i}, y^{i}\right)$ the standard local coordinate system in $T M$.

A spray $\mathbf{G}$ on $M$ is a smooth vector field on $T M_{0}$ expressed in a standard local coordinate system $\left(x^{i}, y^{i}\right)$ in $T M$ as follows

$$
\mathbf{G}=y^{i} \frac{\partial}{\partial x^{i}}-2 G^{i} \frac{\partial}{\partial y^{i}}
$$

where $G^{i}=G^{i}(x, y)$ are the local functions on $T M$ satisfying $G^{i}(x, \lambda y)=$ $\lambda^{2} G^{i}(x, y)$ for $\forall \lambda>0$. Put

$$
N_{j}^{i}=\frac{\partial G^{i}}{\partial y^{j}}
$$


These are called the nonlinear connection coefficients of G. Set $\omega^{i}=d x^{i}$ and $\omega^{n+i}:=d y^{i}+N_{j}^{i} d x^{j}$. The connection 1-forms of Berwald connection are given by

$$
\omega_{j}^{i}:=\Gamma_{j k}^{i} d x^{k}
$$

where

$$
\Gamma_{j k}^{i}=\frac{\partial N_{j}^{i}}{\partial y^{k}}=\frac{\partial^{2} G^{i}}{\partial y^{j} \partial y^{k}}
$$

We have

$$
d \omega^{i}=\omega^{j} \wedge \omega_{j}^{i} .
$$

The curvature 2-forms of the Berwald connection are defined by

$$
\Omega_{j}^{i}=d \omega_{j}^{i}-\omega_{j}^{l} \wedge \omega_{l}^{i}
$$

Express

$$
\Omega_{j}^{i}=\frac{1}{2} R_{j k l}^{i} \omega^{k} \wedge \omega^{l}-B_{j k l}{ }^{i} \omega \wedge \omega^{n+l} .
$$

The two curvature tensors $R_{j k l}{ }^{i}$ and $B_{j k l}{ }^{i}$ are called Riemann curvature tensor and Berwald curvature tensor, respectively.

The Riemann curvature $\mathbf{R}_{y}=\left.R_{k}^{i} d x^{k} \otimes \frac{\partial}{\partial x^{2}}\right|_{x}: T_{x} M \rightarrow T_{x} M$ is a family of linear maps on tangent spaces which is defined by

$$
R_{k}^{i}=2 \frac{\partial G^{i}}{\partial x^{k}}-y^{j} \frac{\partial^{2} G^{i}}{\partial x^{j} \partial y^{k}}+2 G^{j} \frac{\partial^{2} G^{i}}{\partial y^{j} \partial y^{k}}-\frac{\partial G^{i}}{\partial y^{s}} \frac{\partial G^{s}}{\partial y^{k}} .
$$

Without much difficulty, one can show that

$$
R_{j k l}^{i}=\frac{1}{3}\left(R_{k \cdot l}^{i}-R_{l \cdot k}^{i}\right) \cdot j
$$

and

$$
R_{k}^{i}=y^{j} R_{j k l}^{i} y^{l} .
$$

Here and hereafter, notation "." denotes the vertical derivatives with respect to $y$. For instance, $f_{\cdot k}=\frac{\partial f}{\partial y^{k}}, \quad f_{\cdot k \cdot l}=\frac{\partial^{2} f}{\partial y^{k} \partial y^{l}}$, etc. By (2.7) and (2.8), we see that the two curvature tensors $R_{k}^{i}$ and $R_{j k l}{ }^{i}$ can represent each other. For this reason, they are all called Riemann curvature tensor if there is no confusion.

The well-known Ricci curvature is defined by

$$
\text { Ric }:=R_{m}^{m}=y^{j} R_{j m l}^{m} y^{l} .
$$

Every Finsler metric $F$ on a manifold induces a spray $\mathbf{G}_{F}=y^{i} \frac{\partial}{\partial x^{i}}-$ $2 G^{i}(x, y) \frac{\partial}{\partial y^{i}}$ with the geodesic coefficients

$$
G^{i}=\frac{1}{4} g^{i l}\left\{\left[F^{2}\right]_{x^{k} y^{l}} y^{k}-\left[F^{2}\right]_{x^{l}}\right\},
$$

where $g^{i j}=\left(g_{i j}\right)^{-1}$. Therefore, every Finsler space is a special spray space. 
Let $\mathbf{G}$ be a spray on an $n$-manifold $M$ and $d V=\sigma d x^{1} \cdots d x^{n}$ a arbitrary volume form. The $S$-curvature $\mathbf{S}=\mathbf{S}_{(G, d V)}$ is defined by

$$
\mathbf{S}:=\frac{\partial G^{m}}{\partial y^{m}}-y^{m} \frac{\partial}{\partial x^{m}}(\ln \sigma)
$$

Using the S-curvature $\mathbf{S}=\mathbf{S}_{(G, d V)}$, one can modify the spray $\mathbf{G}$ to

$$
\hat{\mathbf{G}}=\mathbf{G}+\frac{2 \mathbf{S}}{n+1} \mathbf{Y}
$$

where $\mathbf{Y}=y^{i} \frac{\partial}{\partial y^{i}}$ is the canonical vertical vector field. In local coordinates

$$
\hat{G}^{i}=G^{i}-\frac{\mathbf{S}}{n+1} y^{i} .
$$

\section{Projective Ricci curvature}

Let $\mathbf{G}$ be a spray on an $n$-dimensional manifold $M$. Let $d V$ and $d \tilde{V}$ be volume forms with $d V=e^{-(n+1) f} d \tilde{V}$, where $f=f(x)$ is a scalar function on $M$. The S-curvatures $\mathbf{S}=\mathbf{S}_{(G, d V)}$ and $\tilde{\mathbf{S}}=\mathbf{S}_{(G, d \tilde{V})}$ are related by

$$
\mathbf{S}=\tilde{\mathbf{S}}+(n+1) f_{0}
$$

where $f_{0}:=f_{x^{m}}(x) y^{m}$. By (3.12), we see that $\mathbf{S}_{(G, d V)}=(n+1) \phi_{0}$ if and only if $\mathbf{S}_{(G, d \tilde{V})}=(n+1) \tilde{\phi}_{0}$ with $\phi_{0}=\tilde{\phi}_{0}+f_{0}$.

By (1.2), we have

$$
\begin{aligned}
& \operatorname{PRic}_{(G, d V)}=\boldsymbol{R i c}_{G}+(n-1)\left\{\left[\frac{\mathbf{S}}{n+1}\right]^{2}+\frac{\mathbf{S}_{\mid 0}}{n+1}\right\} \\
& \mathbf{P R i c}_{(G, d \tilde{V})}=\boldsymbol{R i c}_{G}+(n-1)\left\{\left[\frac{\tilde{\mathbf{S}}}{n+1}\right]^{2}+\frac{\tilde{\mathbf{S}}_{\mid 0}}{n+1}\right\},
\end{aligned}
$$

where "|" is the horizontal covariant derivative with respect to G. It follows from (3.13) and (3.14) that

$$
\mathbf{P R i c}_{(\mathbf{G}, d \tilde{V})}=\mathbf{P R i c}_{(\mathbf{G}, d V)}-(n-1)\left\{f_{0 \mid 0}-f_{0}^{2}+\frac{2}{n+1} f_{0} \mathbf{S}\right\},
$$

Theorem 3.1 Let $\mathbf{G}$ be a spray on an n-dimensional manifold $M$. The following are equivalent:

(a) $\mathbf{G}$ is projectively Ricci-flat,

(b) for any volume form $d V$ on $M$ there is a scalar function $f$ on $M$ such that

$$
\operatorname{PRic}_{(G, d V)}=(n-1)\left\{f_{0 \mid 0}-f_{0}^{2}+\frac{2}{n+1} f_{0} \mathbf{S}\right\},
$$


(c) for any volume form $d V$ on $M$ there is a scalar function $f$ on $M$ such that

$$
\boldsymbol{R i c}_{G}=-(n-1)\left\{\Xi_{\mid 0}+\Xi^{2}\right\}
$$

where "|" is the horizontal covariant derivative with respect to $\mathbf{G}, f_{0}=f_{x^{m}} y^{m}$, $\Xi:=\frac{\mathbf{S}}{n+1}-f_{0}$ and $\mathbf{S}=\mathbf{S}_{(G, d V)}$.

Proof: (a) $\Rightarrow$ (b). Assume that for some volume form $d \tilde{V}$,

$$
\operatorname{PRic}_{(G, d \tilde{V})}=0 .
$$

Then by (3.15), for any volume form $d V=e^{-(n+1) f} d \tilde{V}$,

$$
\operatorname{PRic}_{(G, d V)}=(n-1)\left\{f_{0 \mid 0}-f_{0}^{2}+\frac{2}{n+1} f_{0} \mathbf{S}\right\},
$$

(b) $\Rightarrow(\mathrm{c})$. It follows from (3.13) and (3.18) that

$$
\begin{aligned}
\boldsymbol{R i c}_{G} & =(n-1)\left\{f_{0 \mid 0}-f_{0}^{2}+\frac{2}{n+1} f_{0} \mathbf{S}\right\}-(n-1)\left\{\left[\frac{\mathbf{S}}{n+1}\right]^{2}+\frac{\mathbf{S}_{\mid 0}}{n+1}\right\} \\
& =-(n-1)\left\{\Xi_{\mid 0}+\Xi^{2}\right\},
\end{aligned}
$$

where $\Xi=\frac{\mathbf{S}}{n+1}-f_{0}$.

(c) $\Rightarrow($ a). Let $d V$ be an arbitrary volume form on $M$. There is a scalar function $f$ on $M$ such that (3.17) holds.

$$
\mathbf{R i c}=-(n-1)\left\{\Xi_{\mid 0}+\Xi^{2}\right\},
$$

where $\Xi=\frac{\mathbf{S}}{n+1}-f_{0}$ and $\mathbf{S}=\mathbf{S}_{(G, d V)}$. Let $d \tilde{V}=e^{(n+1) f} d V$, we have

$$
\tilde{\mathbf{S}}=\mathbf{S}-(n+1) f_{0}=(n+1) \Xi .
$$

By (3.14), we get

$$
\operatorname{PRic}_{(G, d \tilde{V})}=\operatorname{Ric}_{G}+(n-1)\left\{\Xi^{2}+\Xi_{\mid 0}\right\}=0 .
$$

Q.E.D.

As we have mentioned in the introduction, if a spray $\mathbf{G}$ on an $n$-dimensional manifold $M$ satisfies that $\mathbf{R i c}_{G}=0$ and $\mathbf{S}_{(G, d V)}=(n+1) \phi_{0}$ for some volume form $d V$ and scalar function $\phi$ on $M$, then (3.17) holds. Thus $\mathbf{G}$ is projectively Ricci-flat.

Proof of Theorem 1.4 Let $d V$ be an arbitrary volume form on $M$. By Theorem 3.1. there is a scalar function $f$ on $M$ such that

$$
\boldsymbol{R i c}_{G}=-(n-1)\left\{\Xi_{\mid 0}+\Xi^{2}\right\}
$$


where $\Xi:=\frac{\mathbf{S}}{n+1}-f_{0}$ and $\mathbf{S}=\mathbf{S}_{(G, d V)}$. Assume that $\Xi=\Xi(x, y) \neq 0$ for some non-zero $y \in T_{x} M$. Let $c(t)$ be the geodesic with $c(0)=x$ and $c^{\prime}(0)=y$. By assumption on the completeness, we may assume that $c$ is defined on $R=$ $(-\infty, \infty)$. Let $\Xi(t):=\Xi\left(c(t), c^{\prime}(t)\right)$. Then $\Xi^{\prime}(t)=\Xi_{\mid 0}\left(c(t), c^{\prime}(t)\right)$. It follows from (3.19) that

$$
\Xi^{\prime}(t)+\Xi(t)^{2} \leq 0 \text {. }
$$

Case 1: $\Xi(0)<0$. Let $r:=\sup \{b>0 \mid \Xi(t)<0,0 \leq t<b\} \leq+\infty$. Observe that for $0 \leq t<r$,

$$
\begin{gathered}
{\left[\frac{1}{\Xi(t)}\right]^{\prime} \geq 1 .} \\
\frac{1}{\Xi(t)}-\frac{1}{\Xi(0)} \geq t . \\
0>\frac{1}{\Xi(t)} \geq \frac{1+\Xi(0) t}{\Xi(0)} .
\end{gathered}
$$

This implies that $1+\Xi(0) t>0$ for $0 \leq t<r$. Thus $r \leq-1 / \Xi(0)$ is finite. Then $\Xi(r)=0$ by the definition of $r$. Observe that

$$
\Xi(t) \leq \frac{\Xi(0)}{1+\Xi(0) t} \leq \Xi(0)<0, \quad 0 \leq t<r .
$$

Thus $\Xi(r) \leq \Xi(0)<0$. This is impossible.

Case 2: $\Xi(0)>0$. Let $r=\sup \{b>0 \mid \Xi(t)>0,-b<t \leq 0\}$. By a similar argument for $\Xi(t)$ on $(-r, 0]$, we can show that this is impossible.

Therefore $\Xi=0$. Then it follows from (3.19) that $\mathbf{R i c}_{G}=0$. Q.E.D.

Below is a specific non-trivial example.

Example 3.2 Let $\alpha_{1}=\sqrt{a_{i j} y^{i} y^{j}}$ and $\alpha_{2}=\sqrt{\bar{a}_{i j} y^{i} y^{j}}$ be two Ricci-flat Riemannian metrics on the manifolds $M_{1}$ and $M_{2}$, respectively. Consider the following 4-th root metric

$$
F:=\sqrt[4]{\alpha_{1}^{4}+2 c \alpha_{1}^{2} \alpha_{2}^{2}+\alpha_{2}^{4}}
$$

where $0<c \leq 1$ is a constant. It is a Riemannian metric when $c=1$. This is a Ricci-flat $\left(\mathbf{R i c}_{F}=0\right)$ and Berwald metric on $M:=M_{1} \times M_{2}$. Thus for the Busemann-Hausdorff volume form $d V=d V_{F}$, the $S$-curvature $\mathbf{S}_{(F, d V)}=0$. Therefore $F$ is projectively Ricci-flat.

\section{Randers metrics}

In this section, we will derive the equivalent conditions for a Randers metric satisfying equation 3.17 . 
Let $F=\alpha+\beta$ be a Randers metric on an $n$-dimensional manifold $M$, where $\alpha=\sqrt{a_{i j}(x) y^{i} y^{j}}$ and $\beta=b_{i} y^{i}$. Put

$$
\begin{aligned}
& r_{i j}:=\frac{1}{2}\left(b_{i ; j}+b_{j ; i}\right), \quad s_{i j}:=\frac{1}{2}\left(b_{i ; j}-b_{j ; i}\right), \\
& t_{i j}:=s_{i l} s^{l}{ }_{j}, \quad s_{i}:=b^{j} s_{j i}, \quad t_{i}:=b^{j} t_{j i}=s_{m} s_{i}^{m},
\end{aligned}
$$

where ";" denotes the covariant derivative with respect to Levi-Civita connection of $\alpha$. Through out this paper, we will use "0" to denote the contraction with $y^{i}$. For example, $s_{0}^{m}=s_{j}^{m} y^{j}, t_{00}=t_{i j} y^{i} y^{j}, r_{00}=r_{i j} y^{i} y^{j}$. The geodesic coefficients of $\mathbf{G}=\mathbf{G}_{F}$ are given by

$$
G^{i}=\tilde{G}^{i}+P y^{i}, \quad \tilde{G}^{i}=\bar{G}^{i}+\alpha s_{0}^{i},
$$

where $\bar{G}^{i}$ denotes the spray coefficients of $\alpha$ and $P=\frac{r_{00}-2 \alpha s_{0}}{2 F}$ ([8]). Clearly, $\mathbf{G}=y^{i} \frac{\partial}{\partial x^{i}}-2 G^{i} \frac{\partial}{\partial y^{i}}$ and $\tilde{\mathbf{G}}=y^{i} \frac{\partial}{\partial x^{i}}-2 \tilde{G}^{i} \frac{\partial}{\partial y^{i}}$ in 4.20 are projectively equivalent. Recall that the projective spray $\hat{\mathbf{G}}=\mathbf{G}+\frac{2 \mathbf{S}}{n+1} \mathbf{Y}$ is projectively invariant for a fixed volume form $d V=\sigma(x) d x^{1} \cdots d x^{n}$. Thus the projective Ricci curvature of $(\mathbf{G}, d V)$ is given by

$$
\mathbf{P R i c}_{(G, d V)}=\mathbf{P R i c}_{(\tilde{\mathbf{G}}, d V)}=\mathbf{R i c}_{\tilde{\mathbf{G}}}+(n-1)\left\{\frac{\tilde{\mathbf{S}}_{\mid 0}}{n+1}+\left[\frac{\tilde{\mathbf{S}}}{n+1}\right]^{2}\right\},
$$

where $\tilde{\mathbf{S}}=\mathbf{S}_{(\tilde{G}, d V)}$ is the $S$-curvature of $(\tilde{\mathbf{G}}, d V)$ and "l" denotes horizontal covariant derivative with respect to $\tilde{\mathbf{G}}$.

Proof of Theorem 1.5. We shall calculate each term on the right side of (4.21) as follows. Firstly, we have known that

$$
\mathbf{R i c}_{\tilde{\mathbf{G}}}=\operatorname{Ric}_{\alpha}+\left(2 \alpha s_{0 ; m}^{m}-2 t_{00}-\alpha^{2} t_{m}^{m}\right),
$$

where ";" denotes the covariant derivative with respect to Levi-Civita connection of $\alpha$ and $\operatorname{Ric}_{\alpha}$ is the Ricci curvature of $\alpha$. From $\tilde{G}^{i}=\bar{G}^{i}+\alpha s^{i}{ }_{0}$, it follows that

$$
\begin{aligned}
\tilde{\mathbf{S}} & =\frac{\partial \tilde{G}^{m}}{\partial y^{m}}-y^{m} \frac{\partial}{\partial x^{m}}(\ln \sigma) \\
& =\frac{\partial \bar{G}^{m}}{\partial y^{m}}-y^{m} \frac{\partial}{\partial x^{m}}\left(\ln \sigma_{\alpha}\right)+y^{m} \frac{\partial}{\partial x^{m}}\left(\ln \frac{\sigma_{\alpha}}{\sigma}\right) \\
& =y^{m} \frac{\partial}{\partial x^{m}}\left(\ln \frac{\sigma_{\alpha}}{\sigma}\right) .
\end{aligned}
$$

Here we have used $\frac{\partial \bar{G}^{m}}{\partial y^{m}}-y^{m} \frac{\partial}{\partial x^{m}} \ln \sigma_{\alpha}=0$ and $\frac{\partial}{\partial y^{m}}\left(\alpha s_{0}^{m}\right)=0$, where $\sigma_{\alpha}=$ $\sqrt{\operatorname{det}\left(a_{i j}(x)\right)}$. Denote by $\mu=\frac{1}{n+1} \ln \frac{\sigma_{\alpha}}{\sigma}$ and $\mu_{0}=\mu_{x^{m}} y^{m}=\frac{\partial \mu}{\partial x^{m}} y^{m}$. Then (4.23) can be rewritten by $\tilde{\mathbf{S}}=(n+1) \mu_{0}$. Thus we have

$$
\begin{aligned}
\tilde{\mathbf{S}}_{\mid 0} & =\tilde{\mathbf{S}}_{; 0}-2 \alpha s{ }_{0}^{m} \tilde{\mathbf{S}}_{\cdot m} \\
& =(n+1)\left(\mu_{0 ; 0}-2 \alpha s_{0}^{m} \mu_{x^{m}}\right) .
\end{aligned}
$$


and

$$
\tilde{\mathbf{S}}^{2}=(n+1)^{2} \mu_{0}^{2} .
$$

Plugging (4.22), (4.24) and 4.25) into (4.21) yields

$$
\begin{aligned}
\operatorname{PRic}_{(G, d V)} & =\operatorname{Ric}_{\alpha}+\left(2 \alpha s_{0 ; m}^{m}-2 t_{00}-\alpha^{2} t_{m}^{m}\right) \\
& +(n-1)\left(\mu_{0 ; 0}-2 \alpha s_{0}^{m} \mu_{x^{m}}\right)+(n-1) \mu_{0}^{2} .
\end{aligned}
$$

On the other hand, by (4.20), the $S$-curvature of $(F, d V)$ is given by

$$
\begin{aligned}
\mathbf{S} & =\frac{\partial G^{m}}{\partial y^{m}}-y^{m} \frac{\partial}{\partial x^{m}}(\ln \sigma) \\
& =\frac{\partial \bar{G}^{m}}{\partial y^{m}}+(n+1) P-y^{m} \frac{\partial}{\partial x^{m}}(\ln \sigma) \\
& =y^{m} \frac{\partial}{\partial x^{m}}\left(\ln \sigma_{\alpha}\right)+(n+1) P-y^{m} \frac{\partial}{\partial x^{m}}(\ln \sigma) \\
& =(n+1)\left(\mu_{0}+P\right),
\end{aligned}
$$

where $P=\frac{r_{00}-2 \alpha s_{0}}{2 F}$. Hence by (4.26) and (4.27), equation (3.16) is equivalent to

$$
\begin{aligned}
& \operatorname{Ric}_{\alpha}+\left(2 \alpha s_{0 ; m}^{m}-2 t_{00}-\alpha^{2} t_{m}^{m}\right) \\
+ & (n-1)\left\{\mu_{0 ; 0}-2 \alpha s_{0}^{m} \mu_{x^{m}}\right\}+(n-1) \mu_{0}^{2} \\
= & (n-1)\left\{-f_{0}^{2}+f_{0 ; 0}-2 P f_{0}-2 \alpha s_{0}^{m} f_{x^{m}}+\frac{2}{n+1} f_{0}\left[(n+1)\left(\mu_{0}+P\right)\right]\right\} \\
= & (n-1)\left\{-f_{0}^{2}+f_{0 ; 0}-2 \alpha s_{0}^{m} f_{x^{m}}+2 f_{0} \mu_{0}\right\} .
\end{aligned}
$$

The above equation is actually in the form of

$$
A+\alpha B=0,
$$

where $A$ and $B$ are polynomial in $y$. We see that this equation is valid if and only if $A=B=0$. Then we obtain

$$
\begin{aligned}
\operatorname{Ric}_{\alpha} & =2 t_{00}+\alpha^{2} t_{m}^{m}-(n-1)\left\{\mu_{0 ; 0}-f_{0 ; 0}+\left(\mu_{0}-f_{0}\right)^{2}\right\}, \\
s_{0 ; m}^{m} & =(n-1) s_{0}^{m}\left\{\mu_{x^{m}}-f_{x^{m}}\right\} .
\end{aligned}
$$

Letting $h:=\mu-f$, we complete the proof of Theorem 1.5

Q.E.D.

\section{The Riemannian case}

Let $F=\alpha$ be a Riemann metric and $d V=e^{-(n+1) f} d V_{\alpha}$ be a volume form on $M$. Then $\mathbf{S}_{(G, d V)}=(n+1) f_{; 0}$. By (1.2), we have

$$
\operatorname{PRic}_{(G, d V)}=\mathbf{R i c}_{\alpha}+(n-1)\left\{f_{0 ; 0}+f_{; 0}^{2}\right\} .
$$

Then Theorem 1.5 reduces to the following 
Corollary 5.1 Let $(M, \alpha)$ be an n-dimensional Riemannian manifold and $d V=$ $e^{-(n+1) f} d V_{\alpha}$ a volume form on $M$. Then $\mathbf{P R i c}_{(G, d V)}=0$ if and only if

$$
\mathbf{R i c}_{\alpha}=-(n-1)\left\{f_{0 ; 0}+f_{; 0}^{2}\right\},
$$

This leads to a new notion of weighted Ricci curvature $\mathbf{R i c}_{\alpha}^{f}$ on a Riemannian $n$-manifold $(M, \alpha)$ with a scalar function $f=f(x)$ on $M$ :

$$
\mathbf{R i c}_{\alpha}^{f}:=\mathbf{R i c}_{\alpha}+(n-1)\left\{f_{0 ; 0}+f_{; 0}^{2}\right\} .
$$

This weighted Ricci curvature and its relationship with other geometric quantities of $(\alpha, f)$ deserves further study.

Example 5.2 Let $\left(\mathbb{S}^{n}, \alpha\right)$ be a Riemannian $n$-sphere with constant sectional curvature $\mathbf{K}_{\alpha}=1$. Recall that in this case, there exists a scalar function $\phi$ on $M$ such that $\phi_{0 ; 0}=-\phi \alpha^{2}$, where ";" is covariant derivative with respect to the Riemann metric $\alpha$. Thus, the Ricci curvature $\mathbf{R i c}_{\alpha}$ of $\alpha$ satisfies

$$
\mathbf{R i c}_{\alpha}=(n-1) \alpha^{2}=-(n-1) \frac{\phi_{0 ; 0}}{\phi} .
$$

Set $f=\ln |\phi|$ which is only defined on $\{\phi \neq 0\}$. Then (5.29) can be rewritten by

$$
\mathbf{R i c}_{\alpha}+(n-1)\left\{f_{0}^{2}+f_{0 ; 0}\right\}=0 .
$$

It is the case in Corollary 5.1.

\section{References}

[1] S.-I. Ohta and K.-T. Sturm, Heat flow on Finsler manifolds, Comm. Pure Appl. Math., 62(2009), 1386-1433.

[2] Z. Shen, Differential Geometry of Spray and Finsler Spaces, Kluwer Academic Publishers, 2001.

[3] Z. Shen, Volume comparison and its applications in Riemann-Finsler geometry, Advances in Math. 128(1997), 306-328.

[4] Z. Shen, Lectures on Finsler geometry, World Scientific Publishers, 2001.

[5] Z. Shen, On sprays with vanishing $\chi$-curvature, preprint, 2019.

[6] S. S. Chern and Z. Shen, RiemannFinsler Geometry, Nankai Tracts in Mathematics, Vol. 6, World Scientific Co., Singapore, 2005.

[7] X. Cheng, Y. Shen and X. Ma, On a class of projective Ricci flat Finsler metrics,Publ.Math. Debrecen. 7528(2017), 1-12. 
[8] X. Cheng and Z. Shen, Finsler Geometry-An Approach via Randers Spaces, Springer and Science Press, New York-Heidelberg-Beijing, 2012.

[9] X. Cheng and Z. Shen, Randers metrics with special curvature properties, Osaka J. Math. 40(2003), 87-101.

[10] X. Cheng and Z. Shen, A class of Finsler metrics with isotropic Scurvature, Israel J. Math. 169(2009), 317-340.

Zhongmin Shen

Department of Mathematical Sciences,

Indiana University-Purdue University Indianapolis, IN 46202-3216, USA.

zshen@iupui.edu

Liling Sun

Department of Mathematics,

Taiyuan University of Technology Taiyuan, 030024, P.R.China.

sunliling@yeah. net 